\title{
Research on the Decision Making Model of Purchasing Second-hand Car
}

\author{
Wenming Cheng \\ Automotive Engineering institute, Jiangxi University of Technology, Nanchang 330098, China
}

Keywords: Second-hand car consumers; Decision making; Influential factors; Principal component analysis.

\begin{abstract}
In recent years, with China's rapid economic growth the price of cars constantly falls and people's mature idea of consumption as well as higher requirement for life ignite sky rocketing consumption for cars. The second-hand car market, as one of the automotive aftermarkets ushers an unprecedented golden age. Consumers are the key for the survival of second-hand car market, therefore, correctly mastering the basic principle of consumer decision-making, and which factors shall influence their purchasing are helpful to make market strategy and management strategy. This paper firstly reviews relevant researches on consumer decision-making and take second-hand car consumers as the research objectives based on existing research to carry out questionnaire. What's more, based on data analysis on effective questionnaire with Engel's model theory, this paper analyzes five influential factors such as the problem recognition, information search, solution evaluation and selection, purchase decision implementation, and post-purchase behavior to finally come up with the decision making model for second-hand car consumers.
\end{abstract}

\section{Introduction}

In recent years, with the rapid development of economy, people's life standard has been developed and they ask higher requirement on subsidiary products; meanwhile, the demand for cars is increasing and the market is expanding which ignite the upgrading of car output. According to the statistical data of China Association of Automobile Manufacturers, the car sales volume in 2012 is 19.2718 million and 19.3064 million with a $4.63 \%$ and $4.33 \%$ year-on-year growth. Among which, the passenger car output is 15.5237 million and 15.4952 million with a $7.17 \%$ and $7.07 \%$ year-on-year growth; besides, the commercial vehicles output is 3.7481 million and 3.8112 million with a year-on-year falling of $4.71 \%$ and $5.49 \%$. Along with the slow growth of new car sales, the second-hand car market becomes a sunrise industry. According to the data of China Automobile Dealers Association, the second-hand car trading volume (mainly for passenger cars)increased from 252000 in 2000 to 4.7914 million in 2012 with a 19 times growth and the cumulative year-on-year growth is $10.6 \%$ with the transaction amount being 263.626 billion Yuan. The development of second-hand car market can promote the smooth circulation of China's automobile industry and improve our country's economic growth.

The second-hand market becomes more and more mature, and the focus of dealers is no longer the quality of cars but consumers which become the core competence of dealers. The sales volume of second-hand cars is the key for survival and development of dealers and the core of sales is the analysis on decision-making. Actually, dealers have already realized that the key for success is to analyze the decision making. The life quality of people has been developed and consumers' consumption philosophy has been changed. Yet, the consumer thinking is difficult to know, which 
brings challenges for dealers to have better performance. In the market which is dominated by consumers, dealers have to firstly understand the purchasing motives, purchasing behavior and key factors which will affect consumer buying to satisfy consumer's needs so as to make reasonable marketing strategy and to develop competence as well as take a place in the market.

The second-hand car management strategy making has important guiding significance for making enterprise strategies. The core of any market strategy is consumer, and dealers have to consider how can second-car consumers make the decision to buy cars, which channels they can get the information and how can they adjust or reform. Clearly grasping the decision making factors which will influence the purchasing behavior will provide strategic guidance for second-hand car dealers in terms of positioning, pricing and market strategy making.

This paper is carried out under the background that the second-hand car market is expanding and the consumer philosophy becomes mature with strengthen power of consumption. It takes second-hand car consumers as research subjects, and whether consumers will buy as the research purpose so as to further explore the factors that affect will affect consumer decision making to get the conclusion. Based on this, this paper aims to provide references for second-hand car consumers in terms of decision making, management strategy and preferential policies.

\section{Characteristics of second-hand car}

With the accelerating pace of life, consumption philosophy gradually becomes mature and sensible and news cars depreciate in value very quickly and the maintenance cost much. However, a lot of people need the vehicles and second-hand cars become the prior choice for individual and families which are choices for rational consumers. Second-hand cars possess the following features:

The price is relatively affordable. Maybe second-hand cars are not popular, but they are cheap if we take into consideration with the increasing price of oil price, distance from the working place to home. Consumers can use half or more than the half of the money of new cars to buy the one which they like. For many young people or consumers who are newbie, second-hand cars can be regarded as the first choice.

Low discount rate: according to the current rules, once the car is registered, the value declines no matter it has been used or not. In General, the new car is basically devalued by $20 \%$ after one year and $35 \%$ after two years and 50\% after three years which is obvious for premium cars. For those consumers who are not rich, it is a good choice to buy practical vehicle with low discount rates such as Santana, Jetta etc which can be used for many years and dealt with after many years without great losses to eliminate the concern of consumers buying behavior.

Consumers won't feel sad if the cars are being scratched. For many families, cars belong to expendable items, and on the road, there are many people who lack driving experience and driving skills and it is inevitable that there should be bump. If the car is new, simple repairing will cost much and consumers will feel sad. Yet, if the car is second-hand, people may not feel sad if it does not affect the image and driving effect. They will think about repairing if the car cannot work.

The selective space is large: consumers who lack money can choose what they like in terms of economy, power, appearance etc. They can choose various types and brands with less money while the selective space for new cars is relatively small.

It is easy to realize the car dream. With the accelerating pace of life, a dazzling array of people expect to have their own cars and second-hand car maybe the best choice without high price and 
many people can buy the brand they like. What's more, it is convenient to go out for a play or go home, which can help complete the car dream of young consumers.

\section{Relevant theories for consumers' decision making strategies}

Decision making plays an important role in each stage and the decisions of consumers are reflected by models, which is worth exploring. At present, widely used models include Howard-Sheth Model, Nicosia Model, EBK model and Philip Kotler model.

Howard-Sheth Model. Howard and Sheth put forward this model in 1963 which considers consumer buying behavior from four aspects, which are stimulation or input factor, external factor, internal factor, reaction or output factor. This model mainly explains the brand selection behavior. Among which, input factor and external factor can stimulate consumers' buying behavior and affect the internal factor, which is equal to psychological activity. Consumers buy things based on the purchasing experience and certain stimulations as well as the function of various factors. The buying behavior

Nicosia Model. Nicosia Model is issued by Nicosia in 1996 which is made up of four parts: the first part is that entrepreneurs and dealers convey information to consumers via various channels and consumers will form an attitude; the second part means that consumers adopt certain approach to collect information and get evaluation and decision making factors to form buying motives; the third part refers to the condition that consumers are driven by consumer motives who finally take actions based on external stimulation; the fourth part is about the evaluation after purchasing and information feedback. After the purchasing, consumers will tell others the experience in the course of using so as to accumulate experience for others and themselves in terms of buying in the future.

Stimulus-response model of Philip Kotler. Kotler puts forward this model based on the psychological model of recognition-stimulation-reaction to reveal the decision making process. This model reflects the condition that consumers make the choice based on the marketing stimulation and micro environment which is the key of this model and that is to analyze the complete process of decision making and various factors that will influence the decision making process.

\section{Behavioral theory for consumer buying behavior}

The main consumer buying behaviors in western countries have its advantages. Based on above research, we can conclude both the advantages and disadvantages and clearly know that the consumer buying behavior is affected by personal factor, cultural factor, social factor, and marketing factor. Consumers have recognitions and understandings and then form the purchasing willingness. Afterwards, they positively collect information and carry out evaluation plan to ignite purchasing behavior. In addition, their experience is told to other consumers which can be regarded as references.

Personal factors. Personal factors include characteristics, attitudes, life style, and education level. Characteristics refers to the summation of their psychological characteristics which are presented frequently and stably, mainly including interests and hobbies, temperament, personality and ability etc. Personal characteristic is based on genetic factor, which is also formed and developed by social practice. Personal characteristics have significant influences on consumers in terms of product recognition, product information collection, product preferences, product selection, and product purchasing efficiency. These characteristics can stimulate consumers' purchasing requirement and 
motives which will also create great impact on the consumer loyalty and utilization rate with permanent influences.

Cultural factors. Cultural factors refer to the culture, subculture and consumer custom etc. In general, culture refers to the summation of material wealth and spiritual wealth in the social development course including material culture, system culture, and behavior culture. Subculture refers to the culture owned by the secondary group such as religious culture, national culture and regional culture. The means and approaches of consumers collect information are determined by culture and subculture and meanwhile culture determines the selection of consumer subjective consumer behavior which directly affects the consumer experience. Even though, there are different consumption habits and approaches under different environment the emergence and realization of consumer behavior is same. In each stage, the consumption philosophy and consumption approach of consumers shall create great impact on the purchasing behavior which can slow down the change speed of consumer mind and behavior.

Social factors. Political economy, as the superstructure of society is about the right activity of national management. Besides, the political and economic forms will influence the consumption quantity, approach and product acquisition access which can also directly encourage or limit the consumption behavior and consumer desire. The development level of economy directly affects the purchasing ability of people as well as the consumption level and environment. Meanwhile, the economic structure affects the consumption level and quality. The social class is a group which is made up of people who have similar social status which is determined by the education, occupation and income. The different social class leads to different payment means, accepting and processing methods as well as purchasing approaches. Even though, some people present similarity in terms of values, life style and consumption habits, there are different consumption behaviors caused by different economic income, preferences and education background.

Market mixing factor. In each stage of consumption, the consumption behavior is affected by marketing factors. The marketing mix is the basic concept of marketing management among which, product, price, place and promotion are core elements. Product purchasing is inevitably related to product price. Price is one of the factors which directly affect the consumption behavior. While choosing products, they will compare the quality, performance, brand, package etc so as to make the purchasing behavior. Purchasing channels mainly refer to the place, location and acquisition approach and the general approaches are supermarkets, direct marketing and sales promotion etc. The selection of purchasing channel is the main approach to acquire products which has greatly influence on the purchasing satisfaction and cost and sometimes it will also affect the product selection.

\section{Research on the consumer decision making model of second-hand car}

For families or individuals, car purchasing costs much. Even though, second-hand car development in foreign countries has become mature, the development in China is in an exploring stage and consumers will take long time to consider and sometimes they even give up the idea to buy second-hand cars. The construction of second-hand car decision making model can help to test the factors which will affect the consumption behavior. Besides, the verification results can help second-hand car dealers know about the decision making of potential consumers and reduce the time to make a decision. The second-hand car consumer decision making model construction includes the construction approach, construction ideas and influential factors. 
Principal component analysis is a mathematical transformation method, and it changes a group of variables to uncorrelated variables via linear transformation and is ranked in a descending order. In the mathematics transformation, we keep the volume of the total variance and the first variable is the biggest which is known as the first principal component and the second variable is next to the first one and is not related to the first variable and known as the second principal component. And so on, there are $\mathrm{m}$ components for $\mathrm{m}$ variables.

The basic principle of principal components

If there are various variables, we suppose there are $n$ samples and carry out analysis on the influence factor. Besides, each sample has $\mathrm{s}$ indictors then there is $\mathrm{n} \times \mathrm{s}$ data which is shown in the matrix

$x=\left[\begin{array}{cccc}X_{11} & X_{12} & \cdots & X_{1 s} \\ X_{21} & X_{22} & \cdots & X_{2 s} \\ \cdots & \cdots & \cdots & \cdots \\ X_{n 1} & X_{11} & \cdots & X_{n s}\end{array}\right]$

$$
W_{i}=a_{1 j} X_{1}+a_{2 j} X_{2}+\cdots+a_{s j} X_{s}
$$

The principal component analysis takes $\mathrm{s}$ original variable indicators into $\mathrm{m}$ comprehensive new variables $Z_{1}, Z_{2},,, Z_{\mathrm{m}}(\mathrm{m}<\mathrm{s})$,

and the new variables are known as the first, second and $\mathrm{m}$ component which are independent and can represent original indicators.

$$
W_{m}=A_{i} X \quad \text { Which can be recorded as } W_{m}=A_{i} X
$$

Firstly, we figure out the first principal component and then the second component which should be independent and the covariance is 0 and the variance is the biggest. That is to say, the $\mathrm{m}$ principal components should be independent and the first principal component has the biggest variance and so on. Besides, the last variance should be biggest among $s-m+1$ variances. Here, the variance of principal component is $\lambda$ and that is to say $\mathrm{W}$ is the maximum eigenvalue.

The matrix $(\mathrm{m} \leqslant \mathrm{s}): \quad \lambda$ is the matrix composed by the diagonal components of $\lambda_{1}, \lambda_{2}, \lambda_{3} \ldots \lambda_{m}$ and then:

$$
A=\left|a^{(1)},\right| a^{(2)},|\cdots| a^{(m)}, \mid \quad \lambda=\left[\begin{array}{cccc}
\lambda_{1} & 0 & \cdots & 0 \\
0 & \lambda_{2} & \cdots & 0 \\
\cdots & \cdots & \cdots & \cdots \\
0 & 0 & \cdots & \lambda_{m}
\end{array}\right] \lambda
$$

The meaning of each principal component should be analyzed to carry out comprehensive evaluation. The selection of weight can be conducted by the proportion of the eigenvalue of each component to the total eigenvalue. Based on this, we can have the comprehensive grade: $=\frac{\lambda_{1}}{\sum_{j=1}^{p} \lambda_{j}} W_{i 1}+\cdots+\frac{\lambda_{p}}{\sum_{j=1}^{p} \lambda_{j}} W_{i p}$ 
The comprehensive grade of sample $\mathrm{i}$ is

$$
\frac{\lambda_{1}}{\sum_{j=1}^{p} \lambda_{j}} W_{i 1}+\cdots+\frac{\lambda_{p}}{\sum_{j=1}^{p} \lambda_{j}} W_{i p}
$$

\section{Conclusions}

This paper takes second-hand car consumers as the research objectives and is carried out based on relevant theories of decision making. Besides, this paper takes the second-hand car decision making as the real guidance, taking principal components analysis as the analysis tool, combining the theoretical analysis with real investigation. Moreover, it adopts Engel's Model Theory to analyze social factors, marketing factors and consumer factors which may affect the buying behavior. The conclusion is to construct the decision making model of second-hand car which is tested by the principal components analysis. The model comes up with the idea that, it riches the decision making theories, which has important theoretical and practical meaning for the operation of second-hand car market in China.

Main conclusions are summarized as follows. It reviews the definition of consumer buying behavior and gives a new definition to it based on the researches carried out by foreign scholars which is adapted to the consumer buying behavior, namely the consumer decision making policy.

The research on typical decision making model as well as relevant theories helps to explore the factors, which will affect the purchasing behavior. Besides, it designs the questionnaires for second-hand car decision making.

It takes the second-hand car decision making model as the targets to research on the establishment method. Besides, this paper is conducted based on this method. It takes the questionnaires for second-hand car consumers' decision making as the support, combining the Engle Theory Model to construct the decision making model and discuss the influential factors.

\section{Acknowledgements}

This work was financially supported by the key subject building project (vehicle engineering) of Jiangxi University of Technology.

\section{References}

[1] Wang Zhenzhen. Empirical Study on Impacts of Tourism Consumers' Purchase Decision Making Evaluated by China Online Tourism [D]. Beijing International Studies University, 2012

China Travel online reviews s

[2] Xiaoli. Consumer Behavior [M]. Beijing: China Agricultural University Press, 2011:46 77

[3] Shi Yongdong, Hu Shuhua. Buying Behavior Model and Evaluation on Cars [J]. Auto Industry Research, 2003, (02)

[4] Hong Bo. Analysis on the Consumer Decision Making Model [J]. Journal of Yunnan University of Finance and Economics: Social Science Edition, 2006 (05).

[5] Peng Wei, Deng Desheng. The Application of Clustering Analysis in Consumer Buying Behavior [J]. China Collective Economy, 2009 (01): 90 93 
[6] Lin Shiwen, Sun Yanzhe. AHP Analysis on the Factors Influencing Second-hand Car Purchasing [J]. Shanghai Automotive, 2012,01

[7] Gai Guofeng, Shen Jian. Analysis on the Factors Influencing Second-hand Car Purchasing Behavior [J]. Journal of Jilin Business and Technology College, 2009,9,25（5） : 16-19

[8] Li Meng, Yuan Ye. Assessment on Used Cars [M]. Beijing: Beijing Institute of Technology Press, 2010

[9] Wang Huidong. Empirical Research on the SUV Buying Behavior [D]. Master Dissertation of Tianjin University, 2010

[10] Ding Lideng, Xi Min. Judging the Business Model and Evaluation System of Second-hand Car based on Consumer Behavior [J]. Technology Entrepreneurship, 2008 (1). 